\title{
Stable Propagation of Activity Pulses in Populations of Spiking Neurons
}

\author{
Werner M. Kistler \\ kistler@anat.fgg.eur.nl \\ Swiss Federal Institute of Technology Lausanne, 1015 Lausanne EPFL, Switzerland, \\ and Neuroscience Institute, Department of Anatomy, FGG, Erasmus University \\ Rotterdam, 3000DR Rotterdam, The Netherlands

\section{Wulfram Gerstner} \\ wulfram.gerstner@epfl.ch \\ Swiss Federal Institute of Technology Lausanne, 1015 Lausanne EPFL, Switzerland
}

We investigate the propagation of pulses of spike activity in a neuronal network with feedforward couplings. The neurons are of the spikeresponse type with a firing probability that depends linearly on the membrane potential. After firing, neurons enter a phase of refractoriness. Spike packets are described in terms of the moments of the firing-time distribution so as to allow for an analytical treatment of the evolution of the spike packet as it propagates from one layer to the next. Analytical results and simulations show that depending on the synaptic coupling strength, a stable propagation of the packet with constant waveform is possible. Crucial for this observation is neither the existence of a firing threshold nor a sigmoidal gain function-both are absent in our model-but the refractory behavior of the neurons.

\section{Introduction}

Recently, the propagation of sharp pulses of spike activity through various types of neuronal networks has attracted a lot of attention. There are basically two complementary scenarios where a temporally precise transmission of spikes has been investigated in model studies: spatially extended networks with distance-dependent couplings and layered feedforward structures of pools of neurons. Spatially extended networks have properties similar to those of excitable media and exhibit, for example, solitary waves of spike activity (Kistler, Seitz, \& van Hemmen, 1998; Ermentrout, 1998; Bressloff, 1999; Kistler, 2000). Layered feedforward networks, also known as synfire chains (Abeles, 1991), can be seen as a discretized version of the former, where the smooth propagation of a wave of activity is replaced by a discrete transmission of spikes from one layer to the next. Similar to solitary 
waves in spatially extended networks, the transmission function for spikes can produce an attractive fixed point for the shape of the firing-time distribution in each layer. Such a "spike packet" can propagate in a stable way from one layer to the next (Abeles, 1991; Aertsen, Diesmann, \& Gewaltig, 1996; Maršálek, Koch, \& Maunsell, 1997; Gewaltig, 2000; Diesmann, Gewaltig, \& Aertsen, 1999).

Similarly to Abeles (1991) and Diesmann et al. (1999), we consider in this article a chain of $M$ pools of identical neurons with feedforward coupling. Each neuron is described by the spike response model, a generalization of the integrate-and-fire model. The spike train of neuron $i$ is formalized as a sum of $\delta$ functions, $S_{i}(t)=\sum_{f} \delta\left(t-t_{i}^{f}\right)$, where the firing times $t_{i}^{f}$ of neuron $i$ are labeled by an upper index $f$. The membrane potential $u_{i}$ of a given neuron is the linear response to pre- and postsynaptic action potentials,

$$
u_{i}\left(t, \hat{t}_{i}\right)=\sum_{j, j \neq i} \omega_{i j} \int_{0}^{\infty} \mathrm{d} t^{\prime} \epsilon\left(t^{\prime}\right) S_{j}\left(t-t^{\prime}\right)+\eta\left(t-\hat{t}_{i}\right) .
$$

Here, the response kernel $\epsilon$ describes the form of an elementary postsynaptic potential, $\omega_{i j}$ is the synaptic coupling strength, and $\eta$ is a (negative) afterpotential that accounts for the reset of the membrane potential after the last spike at $\hat{t}_{i}=\max \left\{t_{i}^{f} \mid t_{i}^{f}<t, f=1,2, \ldots\right\}$ and for refractoriness (Gerstner \& van Hemmen, 1992; Gerstner, Ritz, \& van Hemmen, 1993; Kistler, Gerstner, \& van Hemmen, 1997). In the absence of synaptic input, $u_{i}=0$ corresponds to the resting potential of the neuron. The influence of the last-but-one and earlier spikes is neglected so that spike triggering can be described by an input-dependent renewal process (Cox, 1962).

Noise is implemented in the model by a stochastic spike-triggering mechanism. New spikes are defined through a stochastic process that depends on the value of the membrane potential. The probability that a spike will occur in the infinitesimal interval $[t, t+\mathrm{d} t)$ is

$$
\operatorname{prob}\{\text { spike in }[t, t+\mathrm{d} t)\}=f\left[u_{i}\left(t, \hat{t}_{i}\right)\right] \mathrm{d} t .
$$

The function $f$ is called escape rate (or hazard function) (Plesser \& Gerstner, 2000). For simplicity, we assume a semilinear dependence of the firing probability and the membrane potential,

$$
f(u)=[u]_{+},
$$

with $[u]_{+}=u$ if $u>0$ and $[u]_{+}=0$ elsewhere. $f(0)=0$ implies that the neuron is not spontaneously active. This completes the definition of our single-neuron model.

If we assume that neuron $i$ has fired its last action potential at time $\hat{t}_{i}$, we can calculate the probability $s_{i}\left(t, \hat{t}_{i}\right)$ that it will "survive" without firing 
until time $t>\hat{t}_{i}$

$$
s_{i}\left(t, \hat{t}_{i}\right)=\exp \left\{-\int_{\hat{t}_{i}}^{t} f\left[u_{i}\left(t^{\prime}, \hat{t}_{i}\right)\right] \mathrm{d} t^{\prime}\right\},
$$

(cf. Cox, 1962; Gerstner, 2000). The probability density for the next firing time is thus

$$
p_{i}\left(t, \hat{t}_{i}\right)=-\frac{\partial}{\partial t} s_{i}\left(t, \hat{t}_{i}\right)=f\left[u_{i}\left(t, \hat{t}_{i}\right)\right] \exp \left\{-\int_{\hat{t}_{i}}^{t} f\left[u_{i}\left(t^{\prime}, \hat{t}_{i}\right)\right] \mathrm{d} t^{\prime}\right\} .
$$

We consider $M$ pools containing $N$ neurons each that are connected in a purely feedforward manner; neurons from pool $n$ project only to pool $n+1$, and there are no synapses between neurons from the same pool. We assume all-to-all connectivity between two successive pools with uniform synaptic weights $\omega_{i j}=\omega / N$. The membrane potential of a neuron $i$ from pool $n+1$ is thus

$$
\begin{aligned}
u_{i}\left(t, \hat{t}_{i}\right) & =\frac{\omega}{N} \sum_{j \in \Gamma(n)} \int_{0}^{\infty} \epsilon\left(t^{\prime}\right) S_{j}\left(t-t^{\prime}\right) \mathrm{d} t^{\prime}+\eta\left(t_{i}-\hat{t}_{i}\right) \\
& =\omega \int_{0}^{\infty} \epsilon\left(t^{\prime}\right) A_{n}\left(t-t^{\prime}\right) \mathrm{d} t^{\prime}+\eta\left(t_{i}-\hat{t}_{i}\right),
\end{aligned}
$$

with $i \in \Gamma(n+1), \Gamma(n)$ the index set of all neurons that belong to pool $n$, and $A_{n}(t)=N^{-1} \sum_{j \in \Gamma(n)} S_{j}(t)$ the population activity of pool $n$. Integration of $A_{n}$ over a short interval of time thus gives the portion of neurons from pool $n$ that fire an action potential during this interval. The coupling strength between two successive pools $\omega$ describes the amplitude of the resulting postsynaptic potential if all neurons in the presynaptic pool would fire synchronously. A single action potential thus produces only weak postsynaptic potentials that, according to equation 1.3, have only a low chance of triggering the neuron.

The spike trains $S_{i}$ and the population activity $A_{n}$ are random variables. Each pool is supposed to contain a large number of neurons $(N \gg 1)$ so that we can replace the population activity $A_{n}$ in equation 1.6 by its expectation value $\bar{A}_{n}$, which is given by a normalization condition (Gerstner, 2000),

$$
\int_{-\infty}^{t} s_{n}(t, \hat{t}) \bar{A}_{n}(\hat{t}) \mathrm{d} \hat{t}=1-s_{n}(t) .
$$

Here, $s_{n}(t)=s_{n}(t,-\infty)$ accounts for those neurons that have been quiescent in the past (i.e., have not fired up to time $t$ ). The strong law of large numbers ensures that the population activity $A_{n}$ converges in probability to $\bar{A}_{n}$ (in 
the weak topology) as the number of neurons in the pool goes to infinity,

$$
\operatorname{prob}\left\{\lim _{N \rightarrow \infty} \int_{-\infty}^{\infty} A_{n}(t) \phi(t) \mathrm{d} t=\int_{-\infty}^{\infty} \bar{A}_{n}(t) \phi(t) \mathrm{d} t\right\}=1,
$$

for any test function $\phi \in \mathcal{C}^{\infty}(\mathbb{R})$ (cf. Lamperti, 1996).

\section{Pulse Propagation}

Simulation studies (Diesmann et al., 1999) and analytic calculations (Gewaltig, 2000) suggest that a pronounced refractory behavior is required in order to obtain a stable propagation of a spike packet from one layer to the next. If neurons were allowed to fire more than once within one spike packet, the number of spikes per packet and thus the width of the packet would grow in each step. Therefore, we use a strong and long-lasting afterpotential $\eta$ so that each neuron can fire only once during each pulse. The survivor function thus equals unity for the duration $\tau_{\mathrm{AP}}$ of the afterpotential; $s_{n}(t, \hat{t})=1$ for $0<t-\hat{t}<\tau_{\mathrm{AP}}$ and $\tau_{\mathrm{AP}}$ being large as compared to the typical pulse width. Let us denote by $T_{n}$ the moment when a pulse packet arrives at pool $n$. We assume that for $t<T_{n}$, all neurons in layer $n$ have been inactive- $A_{n}(t)=0$ for $t<T_{n}$. Differentiation of equation 1.7 with respect to $t$ (and dropping bars in order to keep notation simple) leads to

$$
A_{n}(t)=-\frac{\partial}{\partial t} s_{n}(t)=f\left[u_{n}(t)\right] \exp \left\{-\int_{-\infty}^{t} f\left[u_{n}\left(t^{\prime}\right)\right] \mathrm{d} t^{\prime}\right\},
$$

with

$$
u_{n}(t)=\omega \int_{0}^{\infty} \epsilon\left(t^{\prime}\right) A_{n-1}\left(t-t^{\prime}\right) \mathrm{d} t^{\prime} .
$$

Equation 2.1 provides an explicit expression for the firing-time distribution $A_{n}(t)$ in layer $n$ as a function of the time course of the membrane potential. The membrane potential $u_{n}(t)$ in turn depends on the time course of the activity $A_{n-1}(t)$ in the previous layer, as shown in equation 2.2. Note that both equations are independent of the network size $N$; their derivation, however, relies on the strong law of large numbers so that $N \gg 1$ is implicitly assumed.

Both equations 2.1 and 2.2 can easily be integrated numerically; an analytic treatment, however, is difficult even if a particularly simple form of the response kernel $\epsilon$ is chosen. Following Diesmann et al. (1999), we therefore concentrate on the first few moments of the firing-time distribution in order to characterize the transmission properties. More precisely, we approximate the firing-time distribution $A_{n-1}(t)$ by a gamma distribution and calculatein step $\mathrm{i}$-the zeroth, first, and second moment of the resulting membrane 
potential in the following layer $n$. In step ii, we use these results to approximate the time course of the membrane potential by a gamma distribution and calculate the moments of the corresponding firing-time distribution in layer $n$. We thus obtain an analytical expression for the amplitude and the variance of the spike packet in layer $n$ as a function of amplitude and variance of the spike packet in the previous layer.

In step $\mathrm{i}$, we assume that the activity $A_{n-1}(t)$ in layer $n-1$ is given by a gamma distribution with parameters $\alpha_{n-1}$ and $\lambda_{n-1}$, that is,

$$
A_{n-1}(t)=a_{n-1} \gamma_{\alpha_{n-1}, \lambda_{n-1}}(t)
$$

Here, $a_{n-1}$ is the portion of neurons of layer $n-1$ that contribute to the spike packet, $\gamma_{\alpha, \lambda}(t)=t^{\alpha-1} \mathrm{e}^{-t / \lambda} \Theta(t) /\left[\Gamma(\alpha) \lambda^{\alpha}\right]$ the density function of the gamma distribution, $\Gamma$ the complete gamma function, and $\Theta$ the Heaviside step function with $\Theta(t)=1$ for $t>0$ and $\Theta(t)=0$ else. The mean $\mu$ and the variance $\sigma^{2}$ of a gamma distribution with parameters $\alpha$ and $\lambda$ are $\mu=\alpha \lambda$ and $\sigma^{2}=\alpha \lambda^{2}$, respectively.

The membrane potential $u_{n}(t)$ in the next layer results from a convolution of $A_{n-1}$ with the response kernel $\epsilon$. This is the only point where we have to refer explicitly to the shape of the $\epsilon$ kernel. For simplicity, we use a normalized $\alpha$ function,

$$
\epsilon(t)=\frac{t}{\tau^{2}} \mathrm{e}^{-t / \tau} \Theta(t) \equiv \gamma_{2, \tau}(t)
$$

with time constant $\tau$. The precise form of $\epsilon$ is not important; similar results hold for a different choice of $\epsilon$. In the present context, spikes are mostly triggered during the raising phase of the (excitatory) postsynaptic potential. We therefore set $\tau=1 \mathrm{~ms}$ for fast AMPA-mediated potentials rather than describe the passive membrane time constant, which is about one order of magnitude larger.

We want to approximate the time course of the membrane potential by a gamma distribution $\gamma_{\tilde{\alpha}_{n}, \tilde{\lambda}_{n}}$. The parameters ${ }^{1} \tilde{\alpha}_{n}$ and $\tilde{\lambda}_{n}$ are chosen so that the first few moments of the distribution are identical to those of the membrane potential, that is,

$$
u_{n}(t) \approx \tilde{a}_{n} \gamma_{\tilde{\alpha}_{n}, \tilde{\lambda}_{n}}(t)
$$

with

$$
\int_{0}^{\infty} t^{n} u_{n}(t) \mathrm{d} t \stackrel{!}{=} \int_{0}^{\infty} t^{n} \tilde{a}_{n} \gamma_{\tilde{\alpha}_{n}, \tilde{\lambda}_{n}}(t) \mathrm{d} t, \quad n \in\{0,1,2\}
$$

\footnotetext{
${ }^{1}$ We use a tilde to identify parameters that describe the time course of the membrane potential. Parameters without a tilde refer to the firing-time distribution.
} 
As far as the first two moments are concerned, a convolution of two distributions reduces to a mere summation of their mean and variance. Therefore, the convolution of $A_{n-1}$ with $\epsilon$ basically translates the center of mass by $2 \tau$ and increases the variance by $2 \tau^{2}$. Altogether, amplitude, center of mass, and variance of the time course of the membrane potential in layer $n$ are

$$
\left.\begin{array}{l}
\tilde{a}_{n}=\omega a_{n-1}, \\
\tilde{\mu}_{n}=\mu_{n-1}+2 \tau, \\
\tilde{\sigma}_{n}^{2}=\sigma_{n-1}^{2}+2 \tau^{2},
\end{array}\right\}
$$

respectively. The parameters $\tilde{\alpha}_{n}$ and $\tilde{\lambda}_{n}$ of the gamma distribution are directly related to mean and variance: $\tilde{\alpha}_{n}=\tilde{\mu}_{n}^{2} / \tilde{\sigma}_{n}^{2}, \tilde{\lambda}_{n}=\tilde{\sigma}_{n}^{2} / \tilde{\mu}_{n}$.

In step ii, we calculate the firing-time distribution that results from a membrane potential with time course given by a gamma distribution as in equation 2.5. We use the same strategy as in step $i$, that is, we calculate the first few moments of the firing-time distribution and approximate it by the corresponding gamma distribution,

$$
A_{n}(t) \approx a_{n} \gamma_{\alpha_{n}, \lambda_{n}}(t) \text {. }
$$

The zeroth moment of $A_{n}(t)$ (the portion of neurons in layer $n$ that participates in the activity pulse) can be cast in a particularly simple form; the expressions for higher-order moments, however, contain integrals that have to be evaluated numerically. For amplitude, center of mass, and variance of $A_{n}(t)$, we find

$$
\left.\begin{array}{rl}
a_{n} & =1-\mathrm{e}^{-\tilde{a}_{n}}, \\
\mu_{n} & =m_{n}^{(1)}, \\
\sigma_{n}^{2} & =m_{n}^{(2)}-\left[m_{n}^{(1)}\right]^{2},
\end{array}\right\}
$$

with

$$
\begin{aligned}
m_{n}^{(k)}= & \left(1-\mathrm{e}^{-\tilde{a}_{n}}\right)^{-1} \int_{0}^{\infty} u_{n}(t) \exp \left[-\int_{-\infty}^{t} u_{n}\left(t^{\prime}\right) \mathrm{d} t^{\prime}\right] t^{k} \mathrm{~d} t \\
= & \frac{\tilde{a}_{n} \tilde{\lambda}_{n}^{k}}{\left(1-\mathrm{e}^{-\tilde{a}_{n}}\right) \Gamma\left(\tilde{\alpha}_{n}\right)} \\
& \times \int_{0}^{\infty} \exp \left[-t-\tilde{a}_{n} \Gamma\left(\tilde{\alpha}_{n}, 0, t\right) / \Gamma\left(\tilde{\alpha}_{n}\right)\right] t^{k-1+\tilde{\alpha}_{n}} \mathrm{~d} t
\end{aligned}
$$

being the $k$ th moment of the firing-time distribution (see equation 2.1) that results from a gamma-shaped time course of the membrane potential. $\Gamma\left(z, t_{1}, t_{2}\right)=\int_{t_{1}}^{t_{2}} t^{z-1} \mathrm{e}^{-t} \mathrm{~d} t$ is the generalized incomplete gamma function. The last equality in equation 2.10 has been obtained by substituting $\tilde{a}_{n} \gamma_{\tilde{\alpha}_{n}, \tilde{\lambda}_{n}}(t)$ for $u_{n}(t)$. 
A combination of equations 2.7 and 2.9 yields explicit expressions for the parameters $\left(a_{n}, \mu_{n}, \sigma_{n}\right)$ of the firing-time distribution in layer $n$ as a function of the parameters in the previous layer. The mapping $\left(a_{n-1}, \mu_{n-1}, \sigma_{n-1}\right) \rightarrow$ $\left(a_{n}, \mu_{n}, \sigma_{n}\right)$ is closely related to the neural transmission function for pulsepacket input, as discussed by Diesmann et al. (1999).

Particularly interesting is the iteration that describes the amplitude of the spike packet,

$$
a_{n}=1-\mathrm{e}^{-\omega a_{n-1}}
$$

which is independent of the shape of the spike packet. If $\omega \leq 1$, the mapping $a_{n-1} \rightarrow a_{n}$ has a single (globally attractive) fixed point at $a=0$. In this case, no stable propagation of spike packets is possible since any packet will finally die out. ${ }^{2}$ For $\omega>1$, a second fixed point at $a_{\infty} \in(0,1)$ emerges through a pitchfork bifurcation. The new fixed point is stable, and its basin of attraction contains the open interval $(0,1)$. The fact that the all-off state at $a=0$ is unstable for $\omega>1$ is related to the fact that there is no real firing threshold in our model.

Figure 1 shows examples of the propagation of a spike packet for various synaptic coupling strengths and initial conditions. Theoretical predictions based on equations 2.7 and 2.9 are compared to simulations of a network with $N=1000$ neurons per layer. In each subfigure, a series of bar charts shows the firing-time distribution of neurons from layers $n=0$ to $n=5$. The flow field illustrates the evolution of the amplitude and the width of the spike packet as it propagates from one layer to the next.

In Figure $1 \mathrm{~A}$, a small coupling strength has been chosen $(\omega=1)$ so that iteration 2.11 has only a single fixed point at $a=0$. Therefore, any spike packet will die out whatever is the initial firing-time distribution in layer $n=0$. Figure $1 \mathrm{~B}$ is another example for $\omega=2$. Here, the iteration 2.11 has a stable fixed point at $a \approx 0.80$, and both simulations and theory show that this fixed point corresponds to a stable propagation of spike packets from one layer to the next. Finally, in Figure 1C $(\omega=4)$, we demonstrate that the iteration of $\left(a_{n-1}, \mu_{n-1}, \sigma_{n-1}\right) \rightarrow\left(a_{n}, \mu_{n}, \sigma_{n}\right)$ converges to a mere translation of the spike packet with an approximately fixed waveform. This waveform is globally attractive so that even a weak and broadly tuned initial firing distribution will become sharper and form a narrow spike packet.

\section{Discussion}

Any information processing scheme that relies on the precise timing of action potentials obviously requires a means to transmit spikes without

\footnotetext{
${ }^{2}$ The decay of the activity is exponential in $n$ if $\omega<1$, for $\omega=1$ the decay is polynomial in $n$.
} 
A

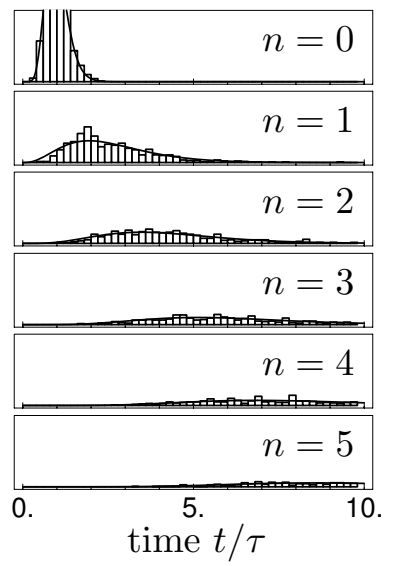

B
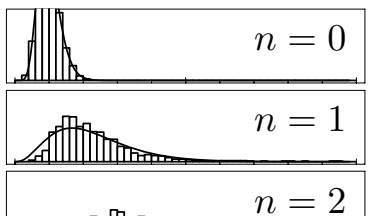

$n=2$

$n=3$

$n=4$

$n=5$

$\begin{array}{rr} \\ 0 . & 5 .\end{array}$

time $t / \tau$

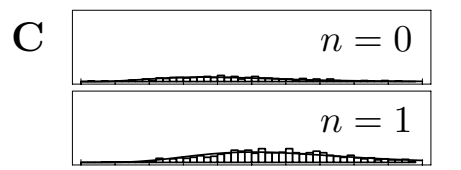

$n=2$

$n=3$

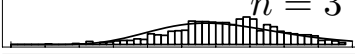

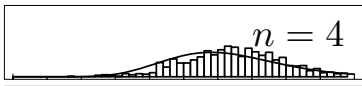

$n=5$

0.

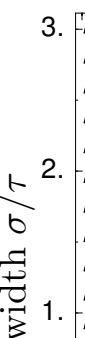

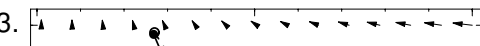

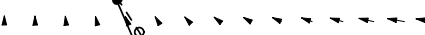

$1+1+1$,

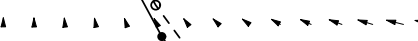

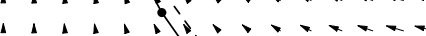

$+1,1,195, \cdots, \ldots$

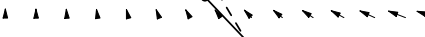

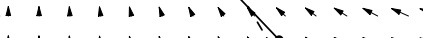

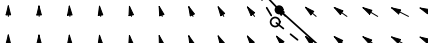

$.1,1,1,1,2$

$1+1+1+1,1$,

$1,1,1,1,1,1,5$

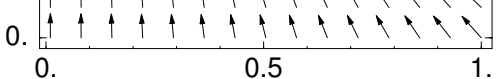

amplitude a
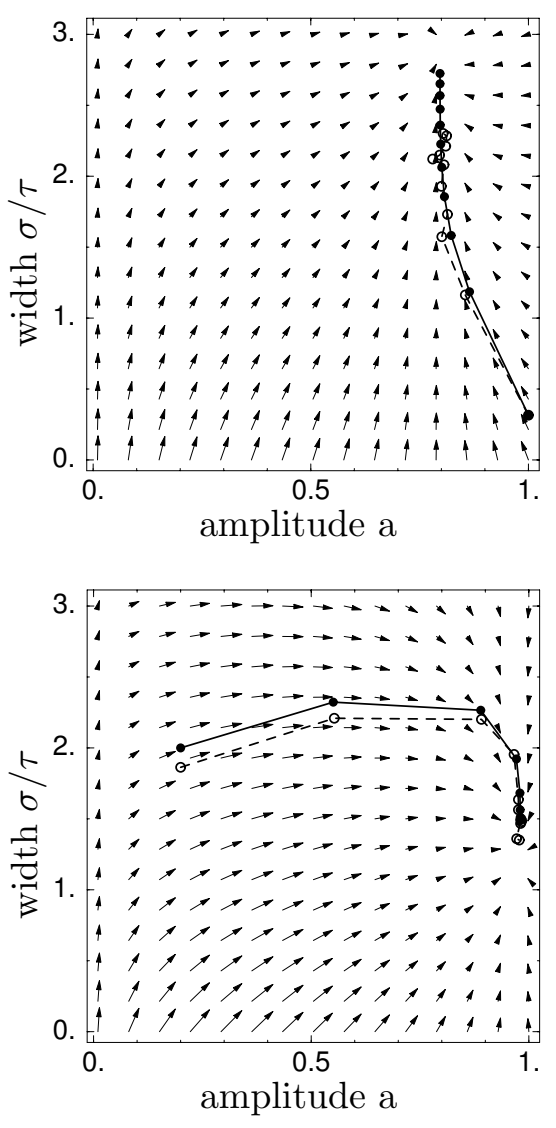
destroying their temporal structure. In this article, we have shown analytically that despite the noise in the spike-generating mechanism, packets of (almost) synchronous spikes can propagate in a feedforward structure from one layer to the next so that their width is preserved, provided that the synaptic coupling strength is sufficiently large.

Our approach is closely related to the concept of synfire-chains (Abeles, 1991). While Abeles stresses the importance of a nonlinear transfer function, ${ }^{3}$ our results are not based on a nonlinear transfer function but are a direct consequence of the refractory behavior of the neurons. Noise and broad postsynaptic potentials tend to smear out initially sharp spike packets. If, however, the synaptic coupling is strong enough, then postsynaptic neurons will start firing during the raising phase of their membrane potential. If, in addition, these neurons show pronounced refractory behavior, then firing will cease even before the postsynaptic potentials have reached their maximum. With respect to precise timing, refractoriness thus counteracts the effects of noise and synaptic transmission.

As a consequence of the linear transfer function, no bistability between asynchronously firing neurons and a propagating pulse could be observed in our model. Depending on the synaptic coupling strength, there is always only one stable fixed point: either the all-off state or a propagating pulse.

Figure 1: Facing page. Propagation of spike packets through a feedforward network. (A) Evolution of the firing-time distribution of a spike packet as it propagates from one layer to the next $(n=0,1, \ldots, 4)$. The neurons in layer $n=0$ are driven by an external input that creates a sharp initial spike packet given by a gamma distribution with $\alpha_{0}=10$ and $\lambda_{0}=0.1$. Initial amplitude is $a_{0}=1$. The bars (bin width 0.2 ) represent the results of a simulation with $N=1000$ neurons per layer; the solid line is the firing-time distribution as predicted by the theory; cf. equations 2.7 and 2.9. The "flow field" to the right characterizes the transmission function for spike packets in terms of their amplitude $a_{n}$ and width $\sigma_{n}=\sqrt{\alpha_{n}} \lambda_{n}$. Open symbols connected by a dashed line represent the simulations shown to the left; filled symbols connected by solid lines represent the corresponding theoretical trajectories. Neurons between layers are only weakly coupled $(\omega=1)$, so that the packet will fade out. Time is given in units of the membrane time constant $\tau$. (B) Same as in $A$ but with increased coupling strength $\omega=2$. There is an attractive fixed point of the flow field at $a=0.80$ and $\sigma=2.9$ that corresponds to the stable waveform of the spike packet. (C) Similar plots as in $A$ and $B$ but with a strong coupling strength $(\omega=4)$. Initial stimulation is weak $\left(a_{0}=0.2\right)$ and broad $\left(\sigma_{0}=2\right)$. As the packet propagates through a few layers, it quickly reaches a stable waveform with amplitude $a=0.98$ and $\sigma=1.5$.

\footnotetext{
${ }^{3}$ The transfer function of Abeles can be retrieved if we replace our equation 1.3 by $f(u) \propto \int_{\vartheta}^{\infty} \rho\left(u^{\prime}-u\right) \mathrm{d} u^{\prime}$ where the membrane potential density $\rho\left(u^{\prime}-u\right)$ is approximated by a gaussian with mean $u$ and a variance $\sigma^{2}$; cf. Abeles (1991, sections 4.5, 7.1-7.3).
} 
This seems to be a severe limitation for the computational usefulness of the system because in the latter case, even a single action potential ultimately can lead to a full-size pulse. Note, however, that this statement holds true only in the limit $N \rightarrow \infty$. Due to the intrinsic probabilistic properties of our model, finite-size effects become important as soon as the activity $A_{n}$ is no longer large as compared to $N^{-1}$. In a finite network, a few initial action potentials will lead to a full-size pulse only with a certain probability smaller than one depending on the size of the network and the distance from the bifurcation. Recent simulation studies (Diesmann et al., 1999) have confirmed that a slightly more general model with a nonlinear transfer function can indeed exhibit bistability where neurons are either firing asynchronously at a low rate or participating in the transmission of a sharp spike packet.

\section{References}

Abeles, M. (1991). Corticonics. Cambridge: Cambridge University Press.

Aertsen, A., Diesmann, M., \& Gewaltig, M.-O. (1996). Propagation of synchronous spiking activity in feedforward neural networks. L Physiol. Paris, 90, 243-247.

Bressloff, P. C. (1999). Synaptically generated wave propagation in excitable neural media. Phus. Rev. Lett., 82, 2979-2982.

Cox, D. R. (1962). Renewal theory. London: Methuen.

Diesmann, M., Gewaltig, M.-O., \& Aertsen, A. (1999). Stable propagation of synchronous spiking in cortical neural networks. Nature, 402, 529-533.

Ermentrout, B. (1998). The analysis of synaptically generated traveling waves. L. Comput. Neurosci., 5, 191-208.

Gerstner, W. (2000). Population dynamics of spiking neurons: Fast transients, asynchronous states, and locking. Neural Comput., 12, 43-89.

Gerstner, W., Ritz, R., \& van Hemmen, J. L. (1993). Why spikes? Hebbian learning and retrieval of time-resolved excitation patterns. Biol. Cybern., 69, 503-515.

Gerstner, W., \& van Hemmen, J. L. (1992). Associative memory in a network of "spiking" neurons. Network, 3, 139-164.

Gewaltig, M.-O. (2000). Evolution of synchronous spike volleys in cortical networksNetwork simulations and continuous probabilistic models. Doctoral dissertation, Shaker Verlag, Aachen, Germany.

Kistler, W. M. (2000). Stability properties of solitary waves and periodic wave trains in a two-dimensional network of spiking neurons. Phus. Rev.E, 62(6), 8834-8837.

Kistler, W. M., Gerstner, W., \& van Hemmen, J. L. (1997). Reduction of the Hodgkin-Huxley equations to a single-variable threshold model. Neural Comput., 9, 1015-1045.

Kistler, W. M., Seitz, R., \& van Hemmen, J. L. (1998). Modeling collective excitations in cortical tissue. Physica D, 114, 273-295.

Lamperti, J. (1996). Probability: A survey of the mathematical theory. New York: Wiley. 
Maršálek, P., Koch, C., \& Maunsell, J. (1997). On the relationship between synaptic input and spike output jitter in individual neurons. Proc. Natl. Acad. Sci. USA, 94, 735-740.

Plesser, H. E., \& Gerstner, W. (2000). Noise in integrate-and-fire neurons: From stochastic input to escape rates. Neural Comput., 12, 367-384.

Received October 31, 2000; accepted July 30, 2001. 


\section{This article has been cited by:}

1. Igor Franović, Vladimir Miljković. 2009. Percolation transition at growing spatiotemporal fractal patterns in models of mesoscopic neural networks. Physical Review E 79:6. . [CrossRef]

2. S Goedeke, M Diesmann. 2008. The mechanism of synchronization in feed-forward neuronal networks. New Journal of Physics 10:1, 015007. [CrossRef]

3. Kazuya Ishibashi, Kosuke Hamaguchi, Masato Okada. 2008. Sparse and Dense Encoding in Layered Associative Network of Spiking Neurons. Journal of the Physical Society of Japan 76:12, 124801. [CrossRef]

4. Dezhe Z. Jin, Fethi M. Ramazanoğlu, H. Sebastian Seung. 2008. Intrinsic bursting enhances the robustness of a neural network model of sequence generation by avian brain area HVC. Journal of Computational Neuroscience 23:3, 283-299. [CrossRef]

5. Kosuke Hamaguchi, Masato Okada, Kazuyuki Aihara. 2007. Variable Timescales of Repeated Spike Patterns in Synfire Chain with Mexican-Hat ConnectivityVariable Timescales of Repeated Spike Patterns in Synfire Chain with Mexican-Hat Connectivity. Neural Computation 19:9, 2468-2491. [Abstract] [PDF] [PDF Plus]

6. Kazuya Ishibashi, Kosuke Hamaguchi, Masato Okada. 2006. Theory of Interaction of Memory Patterns in Layered Associative Networks. Journal of the Physical Society of Japan 75:11, 114803. [CrossRef]

7. A. N. Burkitt. 2006. A review of the integrate-and-fire neuron model: II. Inhomogeneous synaptic input and network properties. Biological Cybernetics 95:2, 97-112. [CrossRef]

8. Kosuke Hamaguchi, Masato Okada , Michiko Yamana, Kazuyuki Aihara . 2005. Correlated Firing in a Feedforward Network with Mexican-Hat-Type ConnectivityCorrelated Firing in a Feedforward Network with Mexican-Hat-Type Connectivity. Neural Computation 17:9, 2034-2059. [Abstract] [PDF] [PDF Plus] 